\title{
Efficient fractionation of lipids in a multi-product microalgal biorefinery by polymers and IL-based aqueous two-phase systems
}

Catalina A. Suarez Ruiz ${ }^{\mathrm{a}}$, Oriol Cabau-Peinado a , Corjan van den Berga, Rene H. Wijffels ${ }^{\mathrm{a}, \mathrm{b}}$, Michel H.M. Eppink ${ }^{1 \mathrm{a}}$

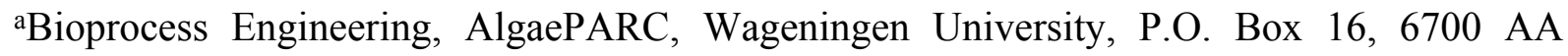
Wageningen, The Netherlands

bNord University, N-8049, Bodø, Norway

Number of pages: 7

Number of tables: 4

Number of figures: 4

${ }^{1}$ Corresponding author: Tel: +31 317482954

Email: michel.eppink@wur.nl (Michel Eppink) 


\section{Supplementary data}

Table S1: Fatty acid composition of individual lipid classes of $N$. oleoabundans. Each value represents the mean of three determinations \pm standard deviation

\begin{tabular}{|c|c|c|c|}
\hline Fatty acid & Name & $\begin{array}{l}\text { PL content } \\
\text { (\% w/w dry matter) }\end{array}$ & $\begin{array}{l}\text { TAG content } \\
\text { (\% w/w dry matter) }\end{array}$ \\
\hline C14:0 & Myristic acid & $0.08 \pm 0.03$ & $0.19 \pm 0.21$ \\
\hline C14:1 cis-9 & Myristoleic acid & 0 & $0.06 \pm 0.05$ \\
\hline C16:0 & Palmitic acid & $2.68 \pm 0.11$ & $6.95 \pm 0.39$ \\
\hline C16:1 & Palmitoleic acid & $0.22 \pm 0.15$ & $0.52 \pm 0.01$ \\
\hline C16:2 & Hexadecadienoic acid & $0.29 \pm 0.12$ & $0.67 \pm 0.04$ \\
\hline C16:3 & Hexadecatrienoic acid & 0 & $0.11 \pm 0.03$ \\
\hline $\mathrm{C} 17: 0$ & Margaric acid & $0.20 \pm 0.07$ & $0.57 \pm 0.01$ \\
\hline C18:0 & Stearic acid & $0.29 \pm 0.11$ & $1.19 \pm 0.08$ \\
\hline C18:1 & Oleic acid & $3.64 \pm 0.15$ & $17.54 \pm 1.05$ \\
\hline C18:2 & Linoleic acid & $2.01 \pm 0.30$ & $4.59 \pm 0.30$ \\
\hline C18:3 & $\alpha$-linolenic acid & $0.44 \pm 0.18$ & $1.14 \pm 0.06$ \\
\hline $\mathrm{C} 20: 0$ & Arachidic acid & 0 & $0.06 \pm 0.05$ \\
\hline C20:1 & Eicosenoic acid & $0.15 \pm 0.25$ & $0.06 \pm 0.02$ \\
\hline $\mathrm{C} 20: 2-\mathrm{n} 6$ & Eicosadienoic acid & $0.001 \pm 0.00$ & 0 \\
\hline $\mathrm{C} 22: 0$ & Behenic acid & $0.08 \pm 0.60$ & 0 \\
\hline \multicolumn{2}{|c|}{ Total content } & $10.07 \pm 0.058$ & $33.64 \pm 0.162$ \\
\hline
\end{tabular}

Table S2: Total fatty acid profile of commercial canola oil

\begin{tabular}{|c|c|c|c|}
\hline Fatty acids & Name & $\begin{array}{l}\text { Oil TAG content } \\
\text { (w/w \%)* }\end{array}$ & $\begin{array}{l}\text { Oil TAG content } \\
(w / w \%)^{a}\end{array}$ \\
\hline C14:0 & Myristic acid & $0.065 \pm 0.000$ & Not available \\
\hline C14:1 cis-9 & Myristoleic acid & 0 & Not available \\
\hline C16:0 & Palmitic acid & $4.547 \pm 0.023$ & 4.2 \\
\hline C16:1 & Palmitoleic acid & $0.290 \pm 0.01$ & Not available \\
\hline C16:2 & Hexadecadienoic acid & $0.066 \pm 0.001$ & Not available \\
\hline C16:3 & Hexadecatrienoic acid & $0.129 \pm 0.002$ & Not available \\
\hline C18:0 & Stearic acid & $1.421 \pm 0.006$ & 2.2 \\
\hline
\end{tabular}




\begin{tabular}{clcc}
\hline C18:1 & Oleic acid & $61.36 \pm 0.11$ & 67.2 \\
C18:2 & Linoleic acid & $19.75 \pm 0.031$ & 18.9 \\
C18:3 & a-linolenic acid & $10.43 \pm 0.06$ & 7.4 \\
C20:0 & Arachidic acid & $0.424 \pm 0.014$ & Not available \\
C20:1 & Eicosenoic acid & $1.265 \pm 0.03$ & 0 \\
C20:2-n6 & Eicosadienoic acid & $0.06 \pm 0.000$ & Not available \\
C22:0 & Behenic acid & $0.194 \pm 0.02$ & Not available \\
\hline
\end{tabular}

*Each value represents the mean of two determinations \pm standard deviation

afrom literature

Table S3: Total fatty acid profile of semi purified polar lipids from yeast

\begin{tabular}{clc}
\hline Fatty acids & \multicolumn{1}{c}{ Name } & $\begin{array}{c}\text { Commercial PL } \\
\text { content }(\mathbf{w} / \mathbf{w} \%)^{*}\end{array}$ \\
\hline C14:0 & Myristic acid & $0.172 \pm 0.172$ \\
C14:1 cis-9 & Myristoleic acid & 0 \\
C16:0 & Palmitic acid & $26.55 \pm 0.413$ \\
C16:1 & Palmitoleic acid & $15.37 \pm 2.06$ \\
C16:2 & Hexadecadienoic acid & 0 \\
C16:3 & Hexadecatrienoic acid & 0 \\
C18:0 & Stearic acid & $24.56 \pm 0.747$ \\
C18:1 & Oleic acid & $19.09 \pm 0.552$ \\
C18:2 & Linoleic acid & $12.33 \pm 1.27$ \\
C18:3 & a-linolenic acid & $1.923 \pm 0.007$ \\
C20:0 & Arachidic acid & 0 \\
C20:1 & Eicosenoic acid & 0 \\
C20:2-n6 & Eicosadienoic acid & 0 \\
C22:0 & Behenic acid & 0
\end{tabular}

*Each value represents the mean of two determinations \pm standard deviation 
(1a)

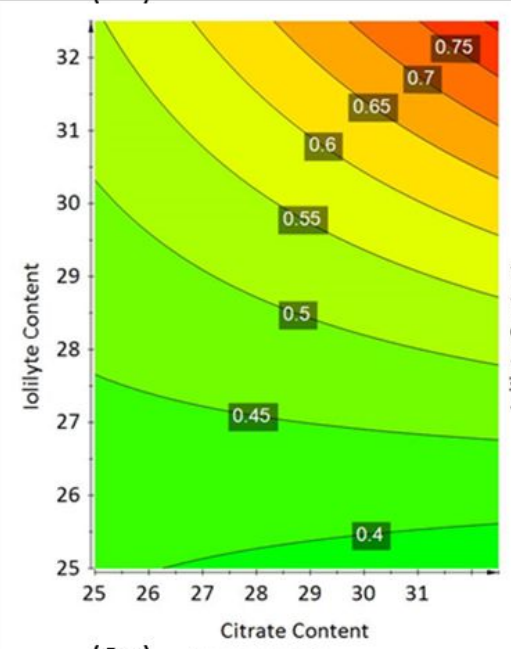

(2a) Temperature $=3$

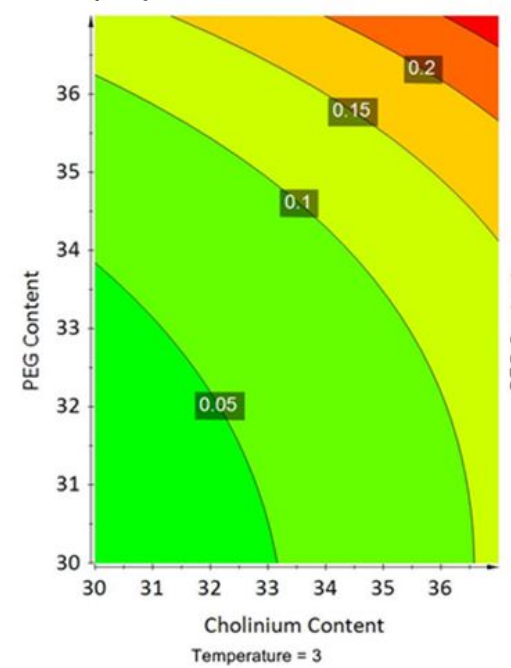

(1b)

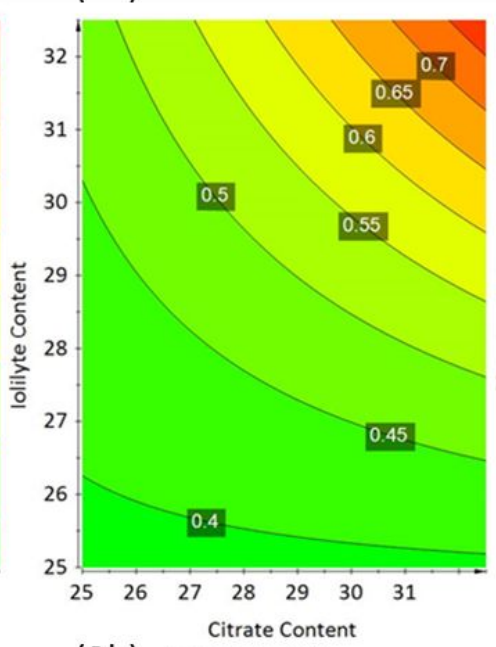

(2b) Temperature $=21.5$

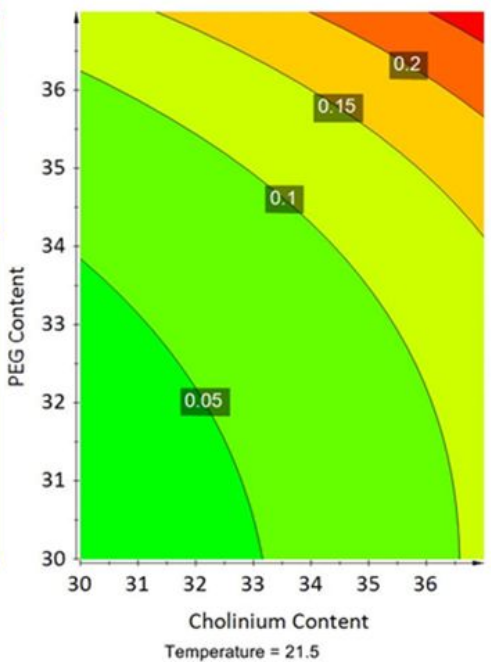

(1c)
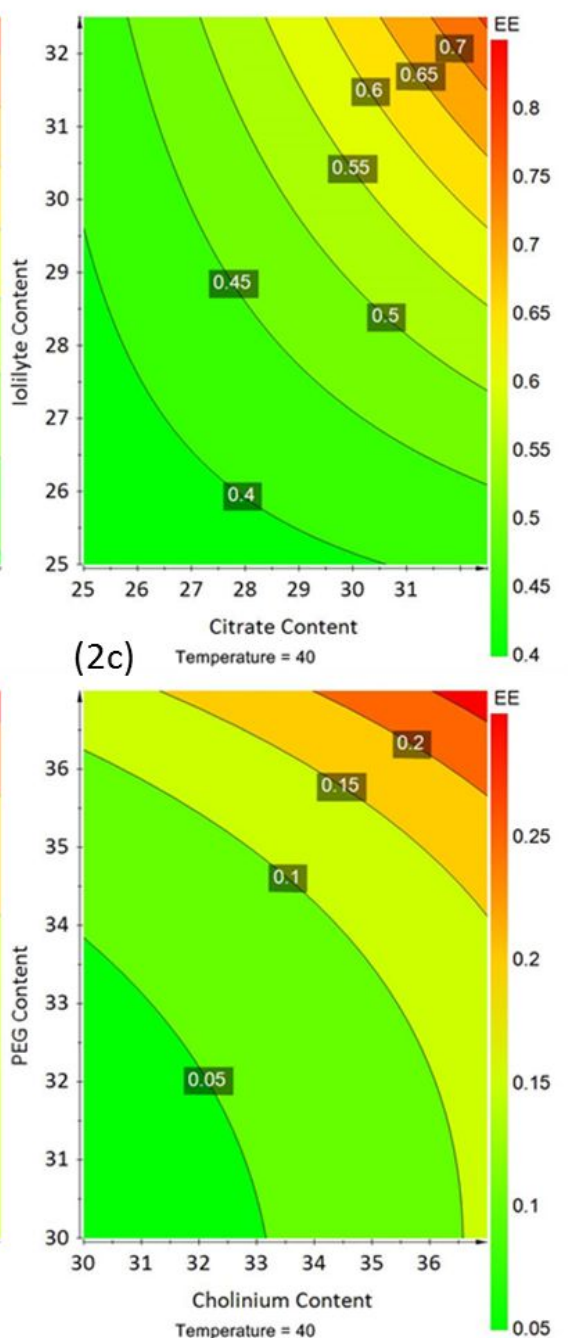

Figure S1: Contour plots showing the influence of the concentration of phase forming components and temperature on the extraction efficiency of TFA from canola oil. ( $1 \mathrm{a}, \mathrm{b}$ and c) Iolilyte 221PG-potassium citrate ATPS; (2 a, b and c) PEG 400-Ch DHp ATPS. 

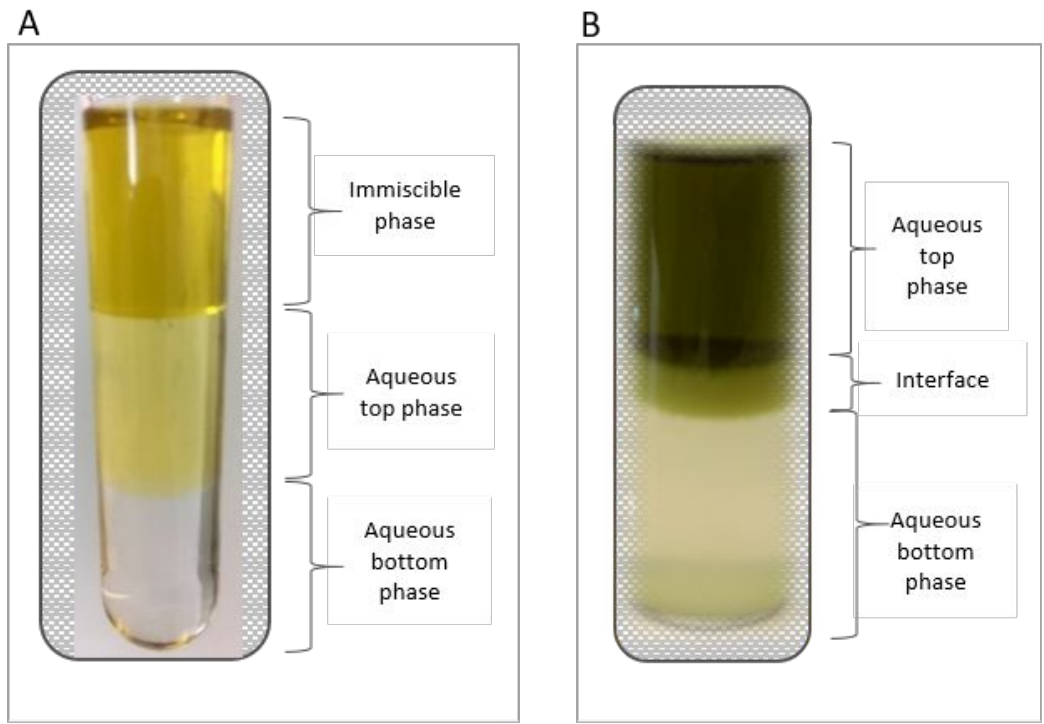

Figure S2: A) Real visual appearance of the partitioning of TAGs from canola oil using PEG 400-Ch DHp ATPS. B) Real visual appearance of the partitioning of TAGs from microalgae using PEG 400-Ch DHp ATPS 
(1a)

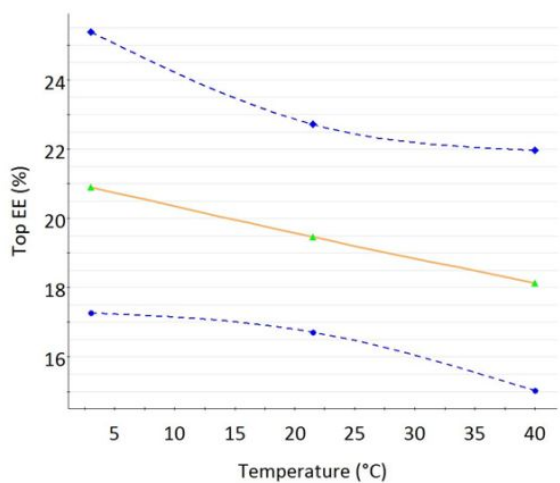

(2a)

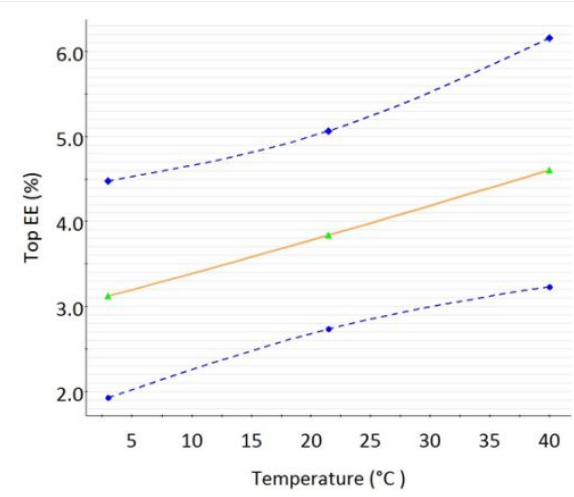

(1b)

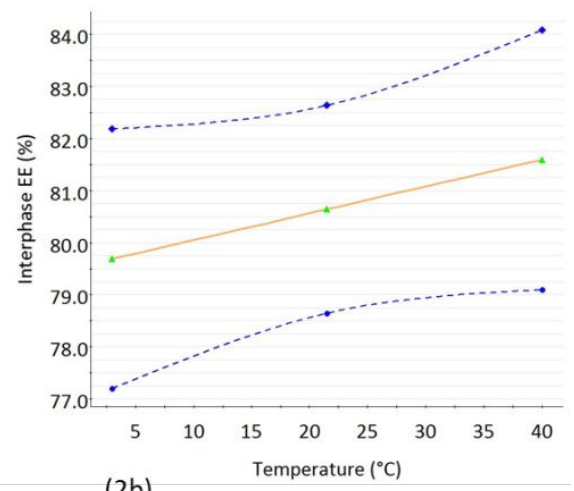

(2b)

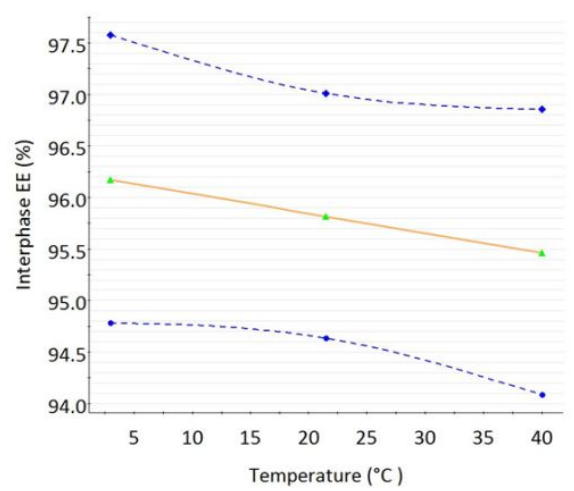

Figure S3: Prediction/Response plot of the effect of temperature on (a) extraction efficiency of TFA from $N$. oleoabundans in the top phase and (b) in the interface using (1) Iolilyte 221PGpotassium citrate ATPS and (2) PEG 400-Ch DHp ATPS. Centre line (orange) shows the calculated response. Top and bottom dashed blue lines show the upper and lower confidence interval (0.95), respectively.

Table S4: Statistical analysis of variance (ANOVA) for the extraction efficiency of TFA from $N$. oleoabundans to the interface using ATPSs.

\begin{tabular}{lcccc}
\hline \multicolumn{1}{c}{ System } & Regression model & Correlation $\left(\mathrm{R}^{2}\right)$ & Variance & Lack of fit \\
\hline $\begin{array}{l}\text { Iolilyte 221PG- } \\
\text { citrate }\end{array}$ & EE \% & 0.92 & 0.005 & 0.347 \\
\hline $\begin{array}{l}\text { PEG 400-Ch } \\
\text { DHp }\end{array}$ & EE \% & 0.92 & 0.006 & 0.110 \\
\hline PEG 400-citrate & EE \% & 0.80 & 0.007 & 0.866 \\
\hline
\end{tabular}

\section{Reduced regression equations}

Iolilyte 221PG-Potassium citrate ATPS 
$\log E E_{\text {int }}=2.5235+0.003342 X_{3}+0.015286 X_{1}^{2}-0.00706 X_{2} X_{3}$

\section{PEG400-Ch DHp ATPS}

$\log E E_{\text {int }}=2.6809-0.00541 X_{1}-0.00673 X_{2}-0.00622 X_{2}^{2}-0.00541 X_{2} X_{3}$

\section{PEG400-potassium citrate ATPS}

$\log E E_{\text {int }}=1.9892-0.0018 X_{2}+0.00511 X_{1}^{2}-0.00167 X_{1} X_{2}$

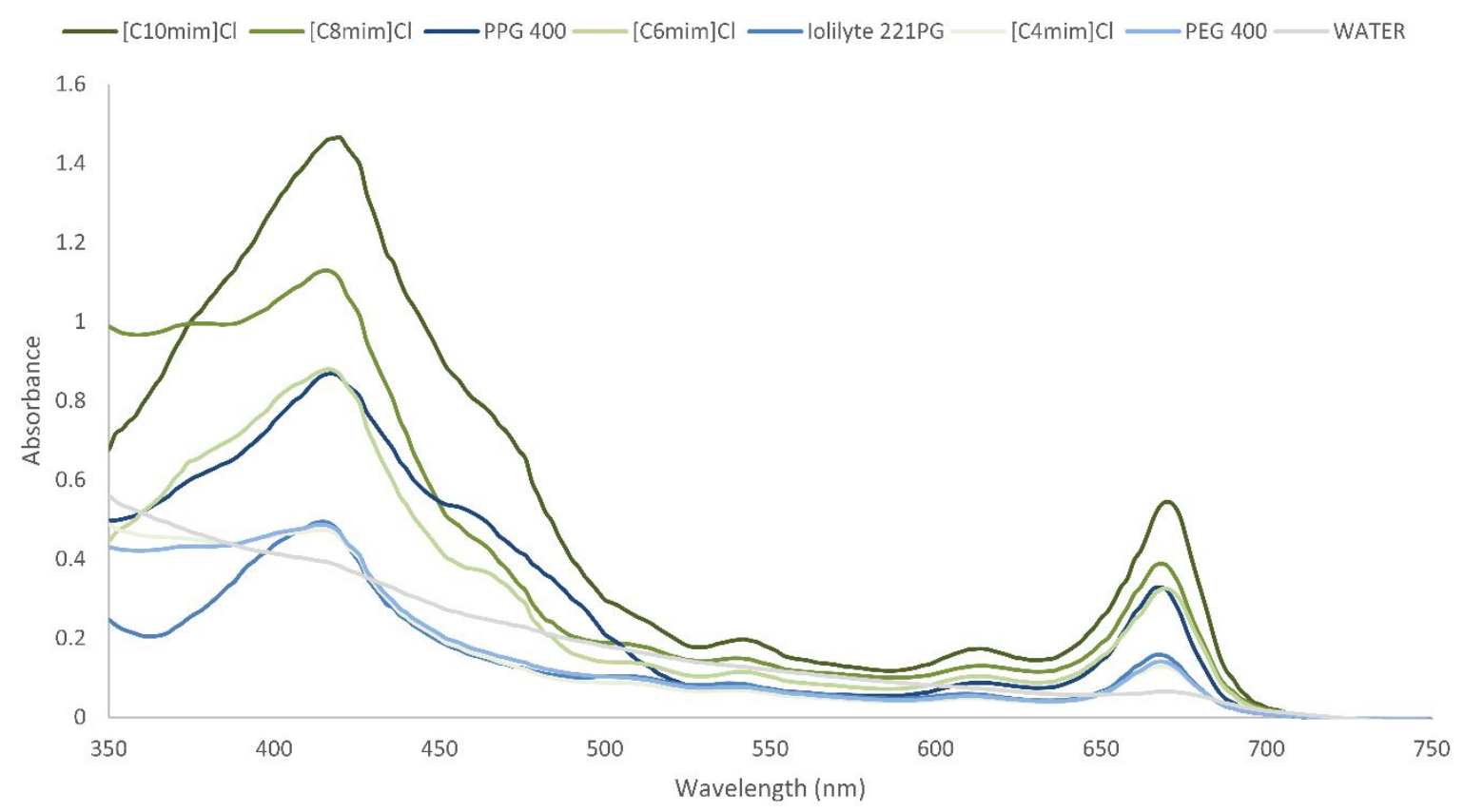

Figure S4: UV-VIS spectra of the extracted pigments using different aqueous solutions. 